\title{
Service-aware LSP selection with fuzzy based packet scheduling scheme for non - real time traffics
}

\author{
Oba Zubair Mustapha ${ }^{1}$, Muhammad Ali², Yim Fun $\mathrm{Hu}^{3}$, Raed A. Abd-Alhameed \\ ${ }^{1}$ Department of Electrical and Electronic Engineering, Institute of Technology, Kwara State Polytechnic, Nigeria. \\ ${ }^{2,3,4}$ Faculty of Engineering and Informatics, University of Bradford, UK.
}

\begin{tabular}{l}
\hline \hline Article Info \\
\hline Article history: \\
Received Oct 23, 2020 \\
Revised Jan 5, 2021 \\
Accepted Mar 25, 2021 \\
\hline
\end{tabular}

Keywords:

Fuzzy logic control Label switched path Multi-protocol label switching Packet scheduling algorithms Traffic engineering

\begin{abstract}
An essential solution is available in Multi-Protocol Label Switching (MPLS), which proffers certain solution to the problems faced in networks of today in terms of bandwidth management, quality-of-service (QoS) availability, and traffic control. This paper is an extension of work on Fuzzy based Packet Scheduling Algorithm (FPSA) combined with Packets Processing Algorithm (PPA) in an Internet Protocol/Multi-Protocol Label Switching (IP/MPLS) networks. This will make provision for an intelligent service to the Label Switched Path (LSP) in MPLS networks. Several research work have been proposed on the MPLS Traffic Engineering. However, it is still imperative to further research on the effect of bandwidth increment on the core network using different mechanisms such as the analytical model of MPLS, expertbased packet scheduling algorithm for MPLS QoS support. Since MPLS do not have provision for intelligent routing, it is necessary to propose an intelligent expert system of FPSA combined with PPA. And analytical model of packet forwarding in the MPLS network would be given to provide suitable solution to traffic congestion and reliable services. Furthermore, the network model created using Network Simulator (NS 2), which carries nonreal time application such as File Transfer Protocol (FTP) with bandwidth variations. The results obtained from trace files are interpreted by AWK script and used for the further analysis. The research on FPSA is necessary based on making the decision for the selection of satisfactory service, whereby solving problem of underutilization and overutilization on the links. However, fuzzy lacks the provision for solving complexity of multiple input parameters.
\end{abstract}

This is an open access article under the $\underline{C C B Y-S A}$ license.

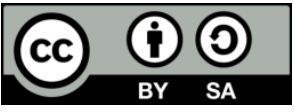

\section{Corresponding Author:}

Oba Zubair Mustapha

Department of Electrical and Electronic Engineering

Institute of Technology, Kwara State Polytechnic

P.M.B 1375, Ilorin Kwara State, Nigeria

Email: obamz2009@gmail.com

\section{INTRODUCTION}

MPLS technology has emerged as an elegant solution to meet the bandwidth-management and service requirements for next-generation of Internet protocol IP-based backbone networks. Furthermore, it is a framework with an Internet Engineering Task Force (IETF) that built an effective routing, switching mechanism and traffic forwarding. This can be used for delivering IP based services and designed to overcome the limitations of IP based forwarding [1]. It ensures the reliability of the communication minimizing the delays and enhancing the speed of packet transfer. It is valuable in its capability of providing Traffic Engineering (TE) for minimizing the congestion by efficient throughput. The flow of packets would suffer long queuing delays at congested nodes and possibly packet loss if buffers overflow. To solve this 
problem, managing the available bandwidth would be of benefit to both the users and operators. An extensive studied and various algorithms of IP-based and MPLS-based Traffic Engineering have been designed and evaluated in [2], [3]. This paper is an extension of work originally presented in [4]. The main purpose of this paper is to perform the evaluation of Label Switched Path (LSP) in IP/MPLS networks with Packet Scheduling Algorithms using NS 2 environment. This is achieved with the incorporation of MPLS network topology and control driven enabled mechanism of Label Distribution Protocol (LDP) in all the MPLS routers [5]. Subsequently, the packet forwarding process is applied, which directed traffic to any route based on explicit route enabled. Then, an output of trace files yielded the results of the simulation

Our contributions to this paper are mention as follows: Firstly, the design of packet scheduling algorithms in IP/MPLS network using NS2 code with input parameters of the non-real time traffics such as sending rate, link bandwidth, propagation delay. This is followed by the analysis of MPLS tables and mathematical model of MPLS operations. Secondly, we increase link bandwidth on packet processing algorithm and fuzzy based packet scheduling algorithm at the interface of core routers. Thirdly, we introduce the fuzzy LSP selection for certain fuzzy rules in the fuzzy logic controller using different fuzzy input variables on the $\mathrm{x}$ and $\mathrm{y}$-axis of the 3-D graph. Fourthly, AWK script is written to interpret the obtained trace files as well as used for the calculation of performance metrics. The remaining parts of this paper commenced in section 2 with the details of MPLS functions and its components as implemented in NS 2. It presents the analytical model of packet mapping and forwarding in the MPLS routers. The proposed Fuzzy based Packet Scheduling Algorithm is provided in the same section. While section 3 elaborates the results with their discussions. Finally, we present our conclusions.

\section{RESEARCH METHOD}

In MPLS, the assignment of a particular packet to a particular Forwarding Equivalence Class (FEC) is done just once, as the packet enters the network. The FEC to which the packet is assigned and encoded as a short fixed length value known as a "label". When a packet is forwarded to its next hop, the label is sent along with it. At subsequent hops, there is no further analysis of the packet's network layer header. Rather, the label is used as an index into a table, which specifies the next hop and a new label is given. The old label is replaced with the new label, and the packet is forwarded to its next hop [6], [5]. It can be observed in the MPLS forwarding paradigm, once a packet is assigned to FEC, subsequent routers do no further header analysis; all forwarding is driven by the labels.

Network simulator (NS) is an IP based simulator where a node consists of classifiers and agents. An agent is the sender/receiver object, while the classifier is the object that is responsible for classifying the arrived packets and then either forwarding them to the convenient nodes or delivering them to the local agent if the receiving node is the packet destination. It uses Tool Command Language (Tcl) for creating a simulation scenario file such as Network topology, transmission time, using protocol. NS consists of event scheduler and IP-based network components. This is written in both Object-oriented Tcl and C++ language. $\mathrm{C}++$ is used for detailed protocol implementation such as packet action and state information management while Tcl is used for simulation configuration such as event scheduling [7]. The following is the steps used to create MPLS network scenarios: Declare simulator; Setting output file; Setting node and link with bandwidth and delay; Setting agents such as Transmission Control Protocol (TCP) and User Datagram Protocol (UDP); Setting applications (FTP) and Constant Bit Rate CBR); Setting simulation time and schedules; Declare finish.

Therefore, the architecture of MPLS Network used in NS2 is illustrated in Figure 1. It consists of four nodes as the sources, seven LSR, and four nodes as the destinations. The incoming packets are allotted with a label by a Label Edge Router (LER) according to their FEC in MPLS [8]. Packets are forwarded alongside with an LSP where each LSR (label switch router) to provide forwarding decision depending on the content of the label, whereby eliminating the need of the IP address so that core router does not have to execute routing lookup, which is very time-consuming process [9]. Folllowing this, the LSR possesses the label leaving the core of each hop and for the next hop, it has to be put on a new label. The decision of packet forwarding can be resolved by next hop by performing the interpretation of the label on the packet of these established paths [10].

The architecture of MPLS shown in [6], [11] allows an LSR to distribute FEC label binding in response to an explicit request from another LSR. NS2 is used to implement the IP/MPLS, which includes the components for the creation of a wired network such as nodes, the link of simplex and duplex type. Each link is configured with the parameters such as bandwidth, propagation delay, and queue type. Data communication between nodes is configured with transport and application layer agents that are required to be attached to both sender and receiver nodes. It also allows an LSR to distribute label bindings to LSRs that have not explicitly requested them [6], [12]. In order for MPLS to operate correctly, label distribution 
information needs to be transmitted reliably, and the label distribution protocol messages pertaining to a particular FEC need to be transmitted in sequence [5]-[13]. The distribution of labels and the construction of LSPs is done by exchanging LDP messages between the LDP agents of LSR nodes as shown in Figure 2.

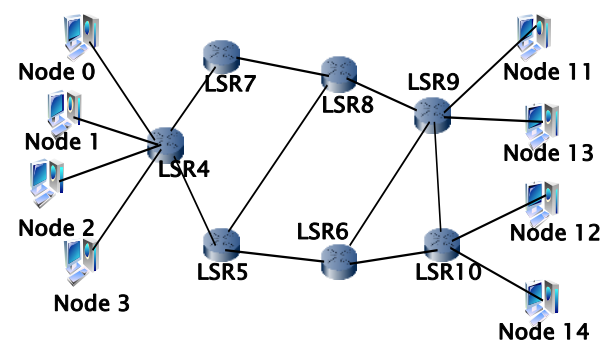

Figure 1. The architecture of MPLS network

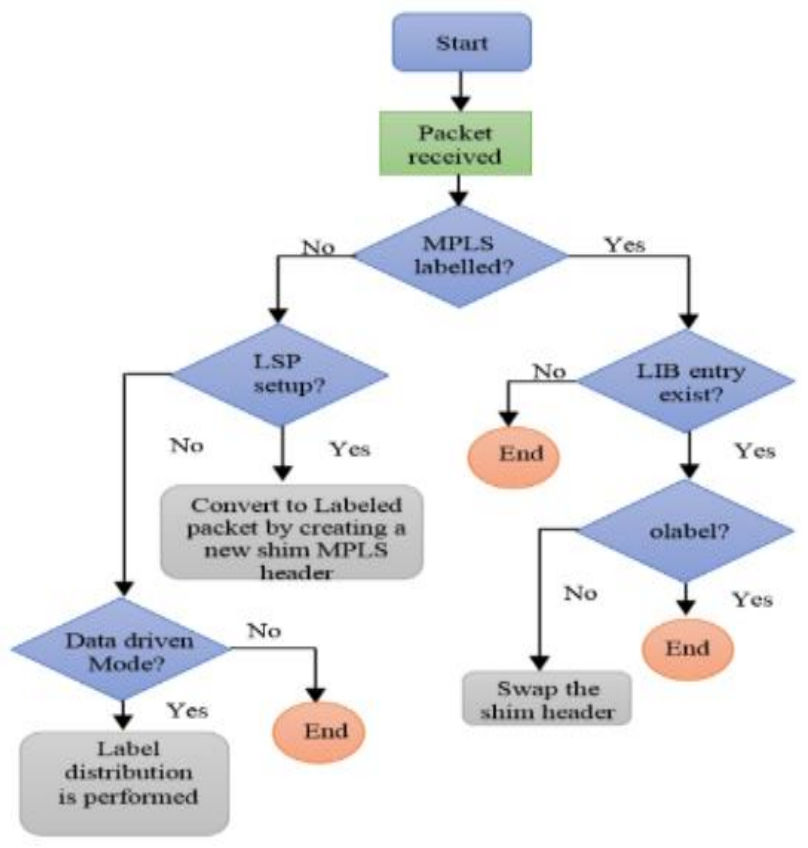

Figure 2. Packet processing algorithm in MPLS

Let $\mathrm{G}=(\mathrm{N}, \mathrm{E})$ be a graph depicting the physical topology of the network. Then, $\mathrm{N}$ is the set of nodes in the network and $\mathrm{E}$ is the set of links; Let $\mathrm{H}=(\mathrm{U}, \mathrm{F}, \mathrm{d})$ be the induced MPLS graph, where $\mathrm{U}$ is a subset of $\mathrm{N}$ representing the set of LSRs in the network, F is the set of LSPs, and d is the set of demands [14]. All the set of routers, in accordance with MPLS network formation, can be categorized into two subsets, In MPLS network, finding a solution to routing issues in terms of flow models is necessary in order to calculate one or a multitude of Label Switching Path (LSP) between a pair of edge "sender-receiver" nodes and define the sequence of the set intensity of traffic distribution between them [15], [16].

$N^{+}=\left\{U_{r}^{+}, r=\overline{1, m_{L E R}}\right\}$ - A Subset of Label Edge Routers (LER).

$N^{-}=\left\{U_{j}^{-}, j=\overline{1, m_{L S R}}\right\}-$ A Subset of Label Switched Routers (LSR).

$U_{r}^{+}-: r-L E R$ at which k-traffic arrives into MPLS Network.

$U_{e}^{+}-: e-L E R$ at which k-traffic leaves MPLS Network.

$K_{r}^{S}$ - : Multitude of $\mathrm{s}$ is in Class of Services $(\mathrm{CoS})$, arriving into $r-L E R$.

$I^{k_{r}^{s}}$-: Intensity of $k_{r}^{s}-$ traffic with servicing class, arriving into $r-L E R$.

$p_{i j}^{k_{r}^{s}}$-: Routing variable, which characterized the intensity of $k_{r}^{s}$ - traffic in $(i, j) \in E$ link for every $r-$ $L E R$ and $k_{r}^{s} \in K_{r}^{s}$. 
$\varphi_{i j}-:$ Intensity of the available link bandwidth from $\mathrm{i}$ to $\mathrm{j}$

$p_{i j}-:$ traffic from $\mathrm{i}$ to $\mathrm{j}$

$$
\left\{\begin{array}{l}
\sum_{J:(i, j) \in E} p_{i j}^{k_{r}^{s}}-\sum_{J:(i, j) \in E} p_{j i}^{k_{r}^{s}}=I^{k_{r}^{s}}, \text { if } i=U_{r}^{+} ; \\
\sum_{J:(i, j) \in E} p_{i j}^{k_{r}^{s}}-\sum_{J:(i, j) \in E} p_{j i}^{k_{r}^{s}}=0, \text { if } i \neq U_{r}^{+}, U_{e}^{+} ; \\
\sum_{J:(i, j) \in E} p_{i j}^{k_{r}^{s}}-\sum_{J:(i, j) \in E} p_{j i}^{k_{r}^{s}}=-I^{k_{r}}, \text { if } i=U_{e}^{+} ;
\end{array}\right.
$$

The equations in (1) imply the number of LERs and LSRs in the network system. Furthermore, it shows the process of packet forwarding in MPLS from the ingress LER (entry) through LSRs to the egress LER (exit). This is to prevent packet loss on the routers in the MPLS network [17], [15]. The whole set of $\mathrm{k}$ traffics, arriving from users (access networks), depending on which edge router this traffic comes from and according to which class it will be serviced.

$$
\sum_{S=1}^{S} \sum_{k_{r}^{s} \in K_{r}^{s}} p_{i j}^{k_{r}^{S}} \leq \varphi_{i j}-\sum_{i=1}^{S} \sum_{\substack{g \in U^{+} \\ g \neq r}} \sum_{k_{r}^{s} \in K_{r}^{s}} p_{i j}^{k_{g}^{s}}\left(r \in U^{+},(i, j) \in E\right)
$$

The meaning of (2) inequality is that the traffic, routed from $r$ - LER, cannot exceed by its intensity of the available bandwidth of the link, which is left after traffics service [7], routed from other edge routers. The parameters related to network information of both non-MPLS and MPLS are shown in Table 1. The list of parameters used in the simulation are as follows: Time of simulation, Number of nodes, Packet size, Sending rate, Interval, Link bandwidth, Source, and end-to-end delay respectively.

Table 1. Simulation parameters

\begin{tabular}{ll}
\hline Parameters & Non-real time traffic \\
\hline Simulation length (s) & 180 \\
Number of nodes & 15 \\
Packet size (bytes) & 1000 \\
Sending rate (kbps) & 500 \\
Interval (s) & 0.005 \\
Link bandwidth (MB) & 2,5 \\
Propagation delay (ms) & 10 \\
Transmission delay (ms) & 4 \\
Processing delay ( $\mu \mathrm{s})$ & $10-20$ \\
Queuing delay (ms) & Variable \\
Type of source & FTP traffic \\
\hline
\end{tabular}

Under the packet forwarding scheme based on the shortest path, packets from node 0 (source) are delivered along LSR 4-7-8-9, and packets from node 1 (source) are delivered along LSR 4-5-6-10. Figure 3 (a) shows the initial simulated network. At $0.5 \mathrm{~s}$ that LDP Mapping Message is used to distribute labels based on control is driven trigger. As a result, every possible LSP in the MPLS network is established [15]. The event at about 34 seconds when flows of FEC 11, FEC 13 and FEC 12, FEC 14 are aggregated into a flow of FEC 10 is illustrated in Figure 3 (b). Subsequently, Figure 3 (c) shows an event at 70 seconds that Constraint-Routing LDP (CR-LDP) request message initiated by LSR4 is delivered along LSR 6-7-8-10 in order to create an ERLSP (Explicit Routing LSP) between LSR4 and LSR10. Figure 3 (d) shows occurrence at 76 seconds that CRLDP Mapping Message is sent by LSR 10 as the response for the LDP Request Message initiated by LSR4.

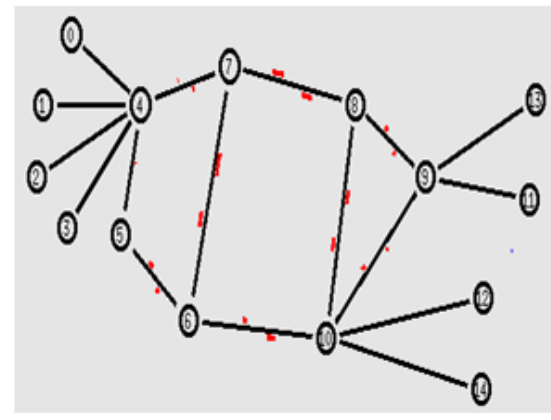

(a)

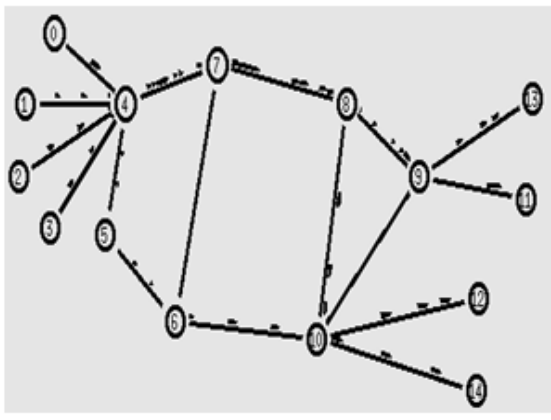

(b) 


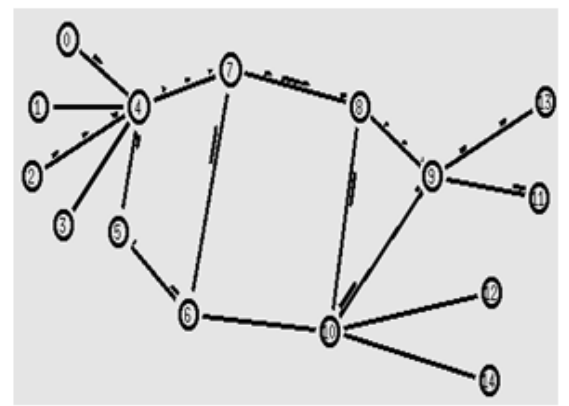

(c)

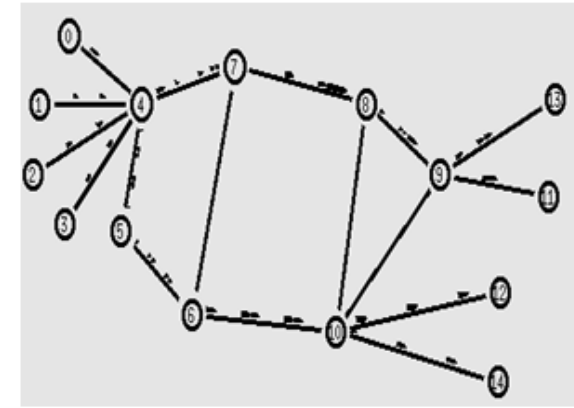

(d)

Figure 3. Label mapping, label distribution, and packet forwarding

\subsection{Application and analysis of fuzzy}

Packet scheduling is the process of deciding which packet to be sent to the outgoing link for transmission. In other words, it is a decision process used to choose which packet to be serviced or dropped. This can influence the delay (and consequently the jitter), bandwidth and loss rate. The most important task of a scheduler is to ensure the satisfaction of QoS requirements for users while efficiently utilizing the available resources. The variants of fair queueing include Weighted Fair Queueing, Stochastic Fair Queueing, and Weighted Round Robin [18]. The proposed fuzzy-based packet scheduling algorithm that would not change the conventional algorithms but make use of operations in order to provide a system that will improve performance. Therefore, Fuzzy Logic Control System (FLCS) is proposed to select paths and then schedule packet into these paths. Since there are many available paths for the same source and destination nodes.

In Conventional Packet Scheduling, Generalized Processor Sharing (GPS) algorithm serves data in queues associated with data flows. Each queue has an associated weight. Weighted Fair Queuing (WFQ) is a packetized version of GPS (PGPS) and tries to simulate GPS on a packet by packet basis [19]. It is a discipline that classified packets by queue. It uses multiple queues to separate flows and gives the flows equal amounts of bandwidth. In this discipline, packets are sorted in weighted order of arrival of the last bit, to determine the transmission order [18]. It is aware of packet sizes and can support variable sized packets, so that flows with large packets are not allocated more bandwidth than the queues with smaller packets. Weighted Round Robin (WRR) is a simple round-robin based scheduling algorithm used in a packet-switch network with the static weight assigned to queues. The queue with higher weight takes the priority to get the process first. It uses variable-sized packets and a deficient counter variable to initialize each flow's weight [19], [20]. It has the limitation of providing a correct percentage of bandwidth to each service class only if all of the packets in queues are of the same size. Deficit Round Robin (DRR) is well-known scheduling originally develop for IP networks. It combines the ability to enable fair queuing in the presence of variable length packets with the simplicity of implementation. It derives ideas from Fair Queuing and Stochastic FQ [10].

This work proposes an intelligent system for packet scheduling in an MPLS network that makes use of fuzzy control. Fuzzy control is based on a relatively simple idea of a fuzzy set that is a generalization of an ordinary set by allowing a degree of membership for each element [21]-[24]. The scheduler [25] can be implemented as a fuzzy controller with inputs such as bandwidth, delay, cost, reliability, packet loss rate, and resources utilization rate. The output defines the LSP that queue from which the next packet will be transmitted. Figure 4 shows the proposed fuzzy logic control system.

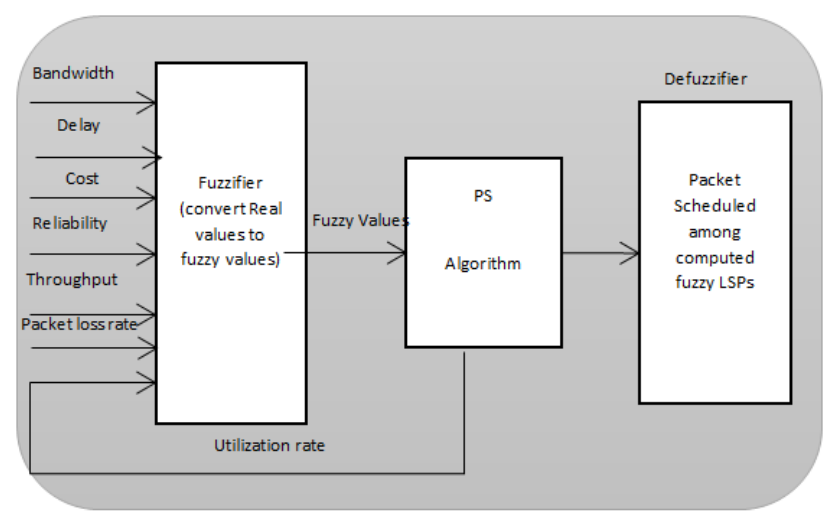

Figure 4. Proposed fuzzy logic control system 
Figures 5 (a) to 5 (g) depict all the degree of membership values with the corresponding crisp input variables. The method of the defuzzification used for this process to get defuzzified output is a weighted average method $\left(\mathrm{z}^{*}\right)$, which implies as in (3):

$\mathrm{Z}^{*}=\frac{\sum \mu(\overline{\mathrm{z}}) \cdot \overline{\mathrm{z}}}{\sum \mu(\overline{\mathrm{z}})}, \overline{\mathrm{z}}=$ assigned weight $; \mu(\overline{\mathrm{z}})=$ membership value

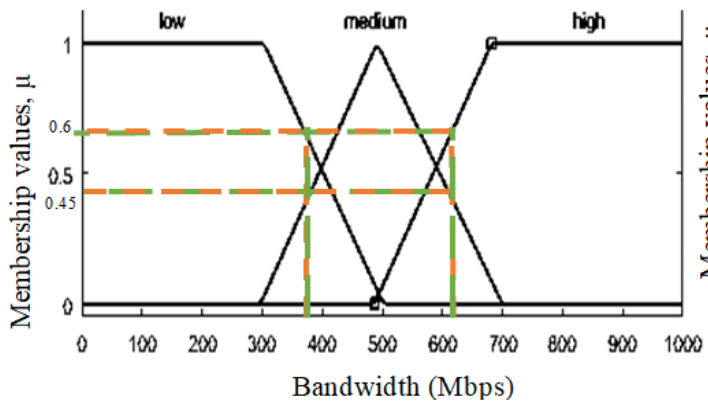

(a)

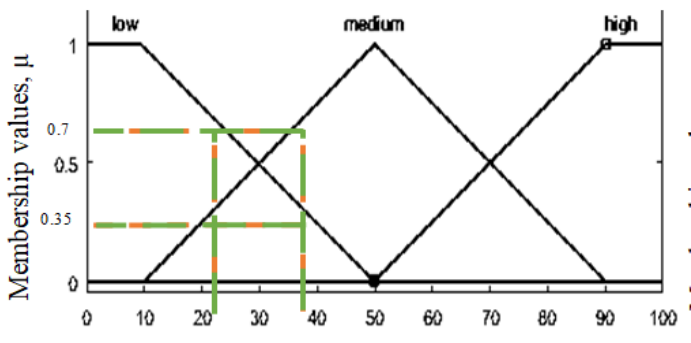

Cost (\%)

(c)

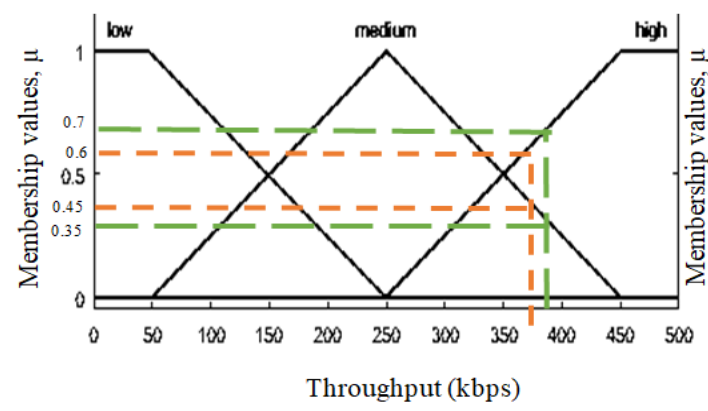

(e)

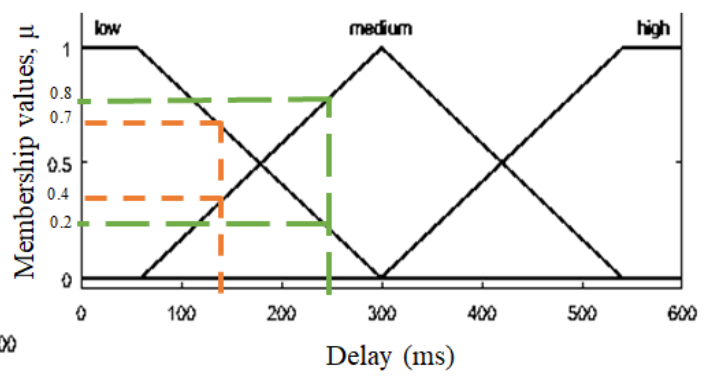

(b)

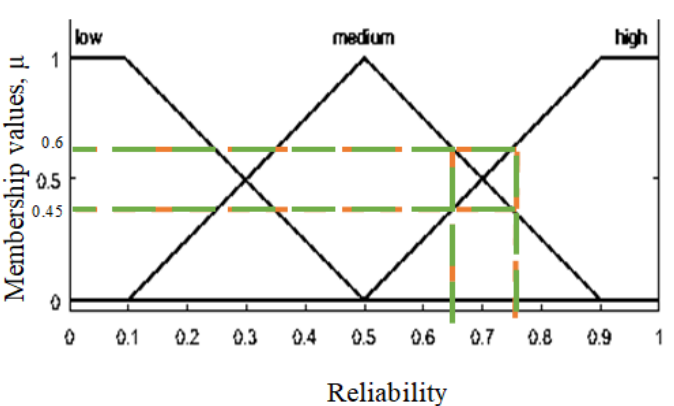

(d)

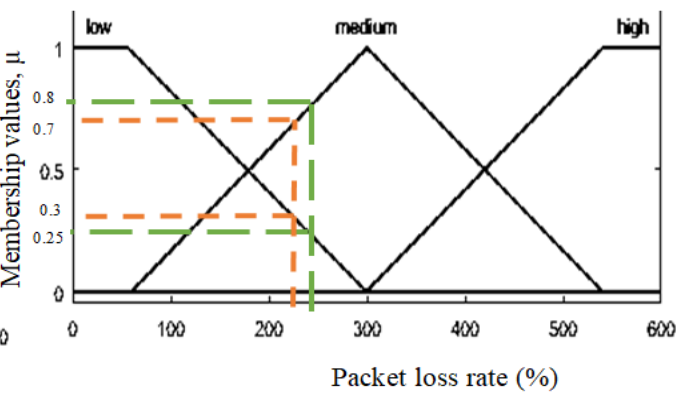

(f)

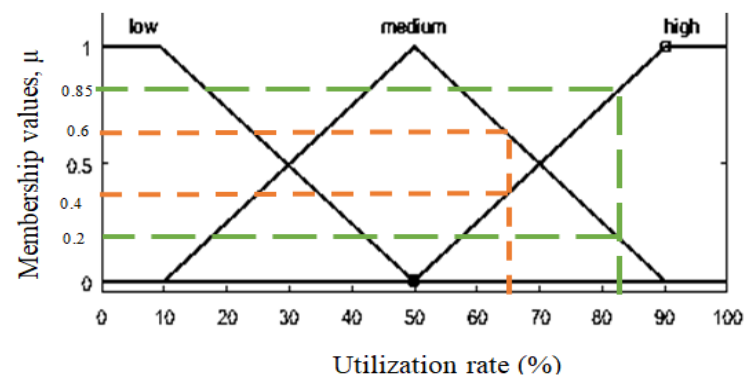

(g)

Figure 5. Membership values of crisp inputs 


\section{RESULTS AND DISCUSSION}

In this section, the results obtained from NS2 implementation of the IP over MPLS with the application of conventional packet scheduling schemes such as First-In-First-Out (FIFO) and Weighted Fair Queuing (WFQ) using non-real time traffics are presented. Then, fuzzy is introduced at the interface of the core routers, which is compared with the operation of MPLS and IP based networks without fuzzy. In Figures 6 and 7, the comparison of average throughput received in IP/MPLS with baseline FIFO and WFQ using FTP are shown. The FTP traffic is created on top of a TCP connection between node 0 and node 10 . It can be seen that MPLS performed better than IP with both algorithms as a result of its fast packet forwarding process through the shortest route. There is an indication of about $43 \%$ increase with WFQ and $49 \%$ increase in the case of FIFO. Furthermore, it is apparent that this figure gives an improved throughput with WFQ in IP/MPLS networks. Similarly, a further improvement is observed as a result of fuzzy applied at the interface of LSR routers. In the first scenario using link bandwidth of $2 \mathrm{MB}$, an increase of about $46 \%$ with WFQ and an approximation of $56 \%$ increment with FIFO are illustrated. In the conventional packet scheduling, approximate value of 35\% increase with WFQ and FIFO with $44 \%$ increment as shown in Figure 8 . The link bandwidth of $5 \mathrm{MB}$ is used in the second scenario with close to $43 \%$ increment for WFQ and about 50\% increase with FIFO as shown in Figure 9. FIFO = Fist In-First Out, WFQ = Weighted Fair Queueing, ftp = file transfer protocol.

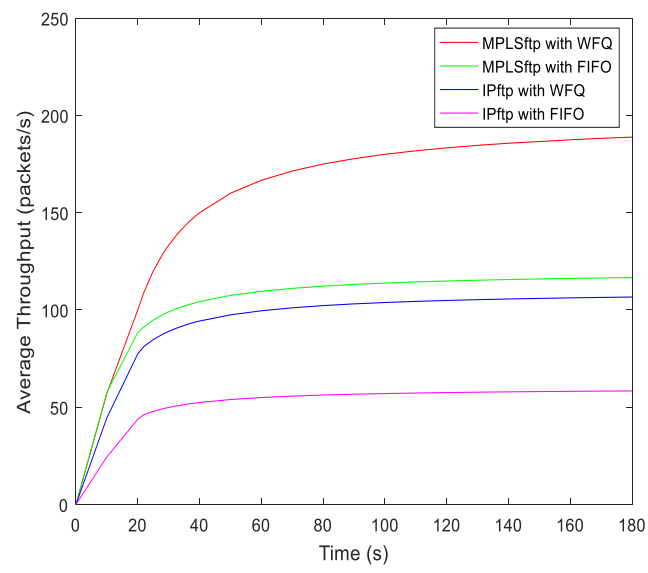

Figure 6. Average throughput of IP/MPLS with baseline FIFO and WFQ using non-real time (without Fuzzy) for $2 \mathrm{MB}$

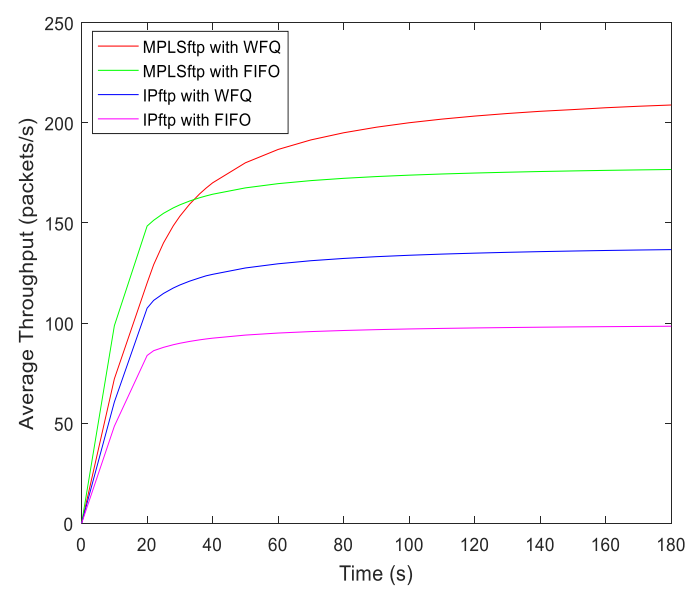

Figure 8. Average throughput of IP/MPLS with baseline FIFO and WFQ using non-real time (without Fuzzy) for $5 \mathrm{MB}$

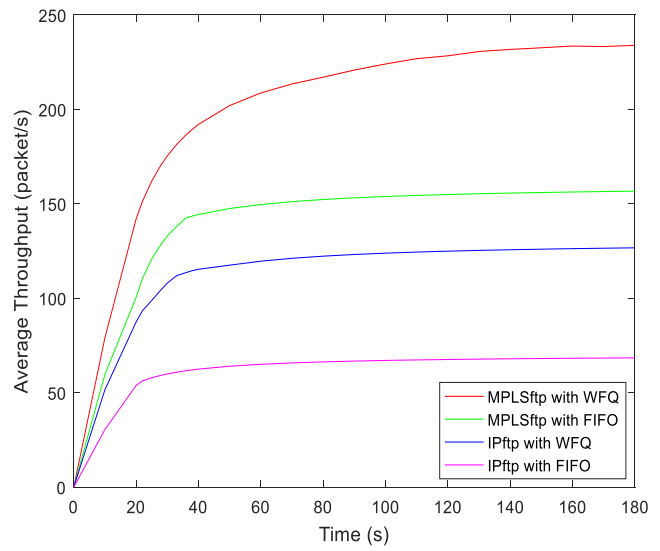

Figure 7. Average throughput of IP/MPLS with baseline FIFO and WFQ using non-real time (Fuzzy) for $2 \mathrm{MB}$

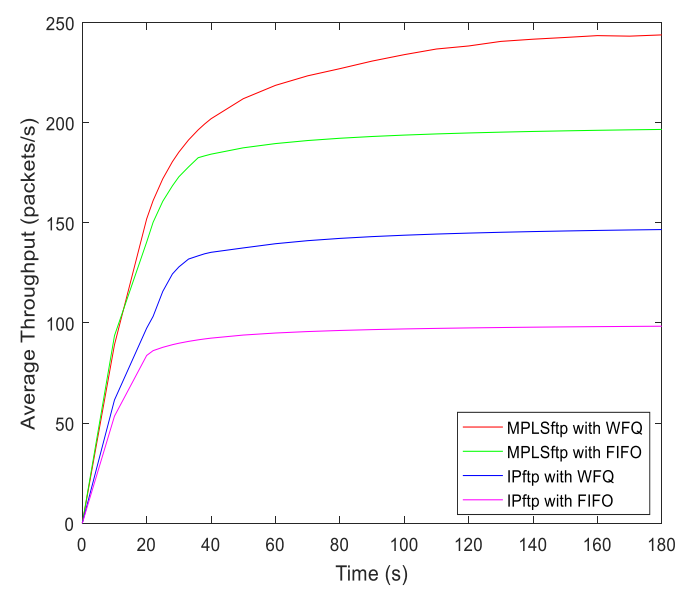

Figure 9. Average throughput of IP/MPLS with baseline FIFO and WFQ using non-real time (Fuzzy) for $5 \mathrm{MB}$ 
Figure 10 shows the impact of average end-to-end delay in the IP/MPLS network with packet scheduling algorithms using a non-real-time application of FTP. A steady delay observed at 1.9 ms with WFQ in MPLS environment as compared with a variation of about $86 \%$ with WFQ in IP network. This implies that WFQ has an absolute low delay than FIFO due to fast packet forwarding mechanism. Furthermore, the delay still has a similar relationship in the performance of FIFO scheduling algorithms within IP and MPLS environments. However, there is small difference as compared with MPLS operations. This is due to the non-sensitive behaviour of FTP applications over TCP. Lower delay is seen after introducing fuzzy to the system in Figure 11. The MPLSftp with WFQ, MPLSftp with FIFO, IPftp with WFQ, and IPftp with FIFO have about $1.3 \mathrm{~ms}, 1.8 \mathrm{~ms}, 5.9 \mathrm{~ms}$, and $7.8 \mathrm{~ms}$ respectively. Therefore, MPLS operations perform better than IP with a scenario of $2 \mathrm{MB}$ of the link bandwidth using conventional and fuzzy-based packet scheduling. Figure 12 and Figure 13 illustrate the performance of IP/MPLS in terms of average end-to-end delay for link bandwidth of $5 \mathrm{MB}$. This is done by comparing the conventional packet scheduling scheme with the fuzzy-based scheme. The average end-to-end delay of WFQ in IP over MPLS and FIFO in IP over MPLS are about $0.1 \mathrm{~ms}, 3.1 \mathrm{~ms}, 0.9 \mathrm{~ms}$, and $3.2 \mathrm{~ms}$, respectively. There is close gap between IP scenarios using FTP application.

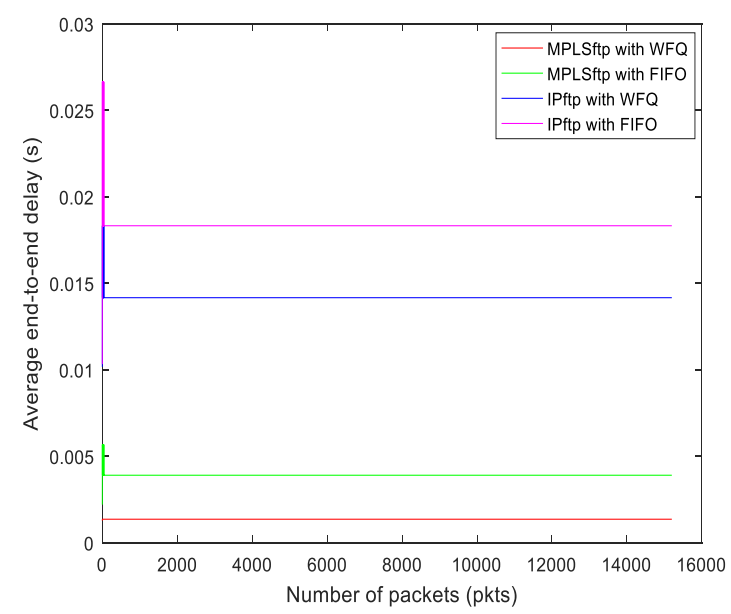

Figure 10. Average end-to-end delay in IP/MPLS with baseline FIFO and WFQ using non-real time (without Fuzzy) for $2 \mathrm{MB}$

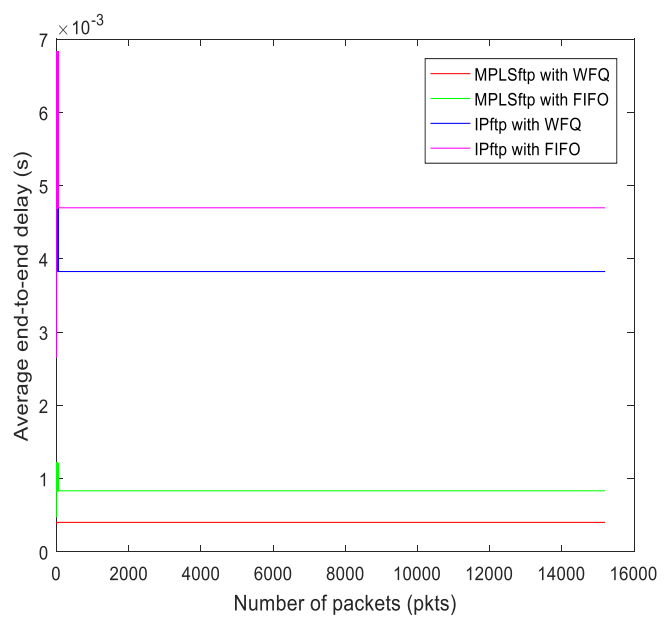

Figure 12. Average end-to-end in IP/MPLS with baseline FIFO and WFQ using non-real time (without Fuzzy) for $5 \mathrm{MB}$

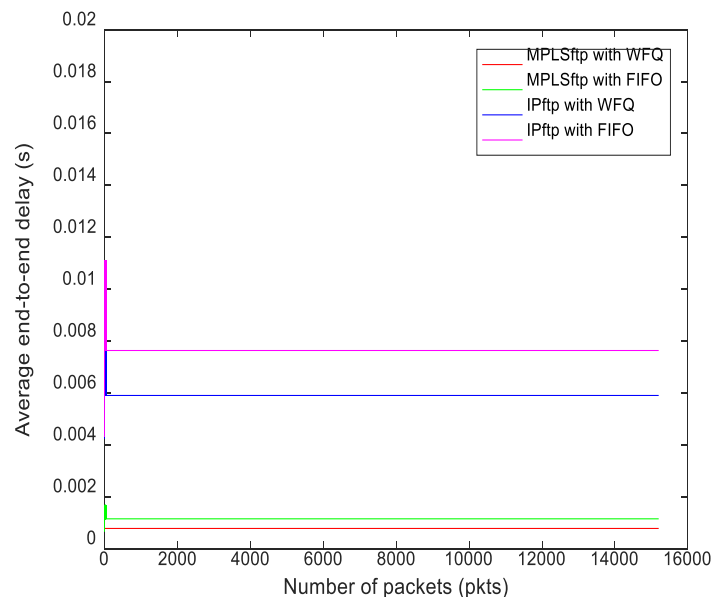

Figure 11. Average end-to-end in IP/MPLS with baseline FIFO and WFQ using non-real time (Fuzzy) for $2 \mathrm{MB}$

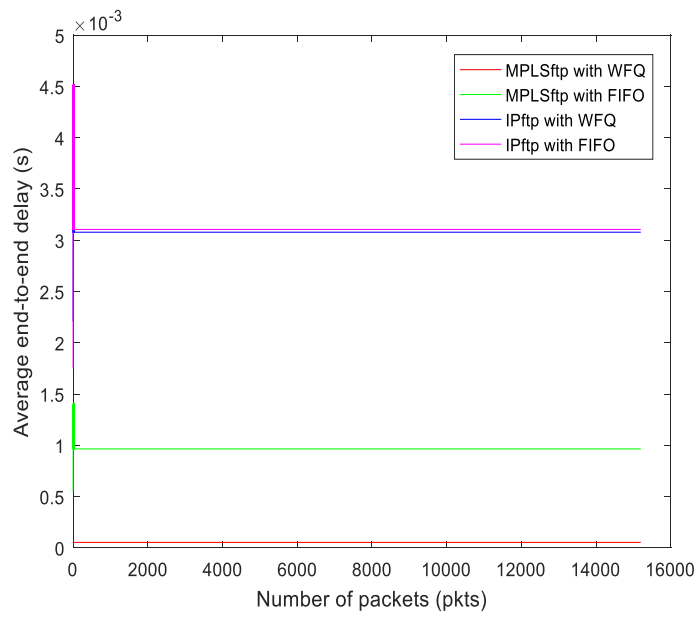

Figure 13. Average end-to-end in IP/MPLS with baseline FIFO and WFQ using non-real time (Fuzzy) for $5 \mathrm{MB}$ 
Figure 14 gives an illustration of a scenario of average queue waiting time in the MPLS network with packet scheduling algorithms using a non-real time application of FTP. It is observed that there is drastic reduction in waiting time due to application of fuzzy to FTP. FIFO reduces by about $38 \%$ while it is $64 \%$ reduction for WFQ. This implies that WFQ has an absolute low waiting than FIFO. Figure 15 shows the reliability for packet delivery in the MPLS network using a non-real time application of FTP. The algorithms of FIFO and WFQ with fuzzy have $66 \%$ and $85 \%$ reliabilities respectively. On the other end, FIFO and WFQ without fuzzy having about $45 \%$ and $56 \%$ reliabilities for packet delivery. The low value of reliability could be as a result of failure in the link, low bandwidth allocation and increase in packet loss.

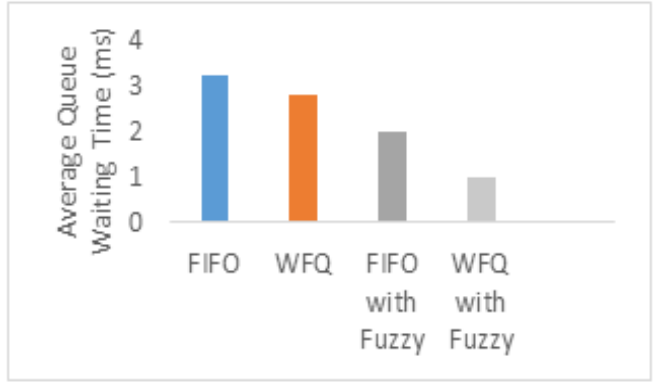

Figure 14. Average queue waiting time for packet scheduling algorithms

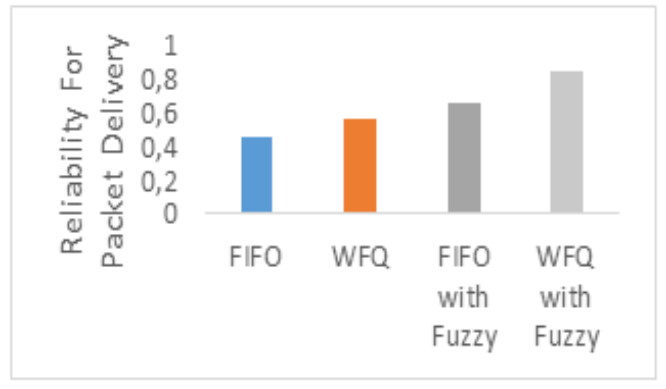

Figure 15. Reliability for packet dleivery using packet scheduling algorithms

The link cost in MPLS network with packet scheduling algorithms using FTP is as shown in Figure 16. The lower the link cost the better for the fast route for the packet forwarding in the core of MPLS network. Fuzzy based algorithms indicate an improve link cost as a result of computation and selection of the lowest link cost. Figure 17 depicts rapid increase in service utilization with the application of fuzzy on WFQ and FIFO scheduling algorithms at the interface of the routers within MPLS environment. However, FIFO and WFQ without fuzzy have lower value of service utilization as compared with an intelligent improved system. This is due to the fast response in packet delivery of file transfer protocol applications, which is served from the source over the transmission control protocol to the receiving end.

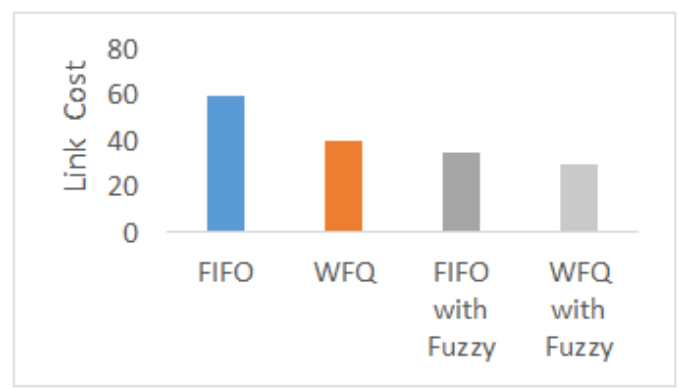

Figure 16. Link cost for packet scheduling algorithms

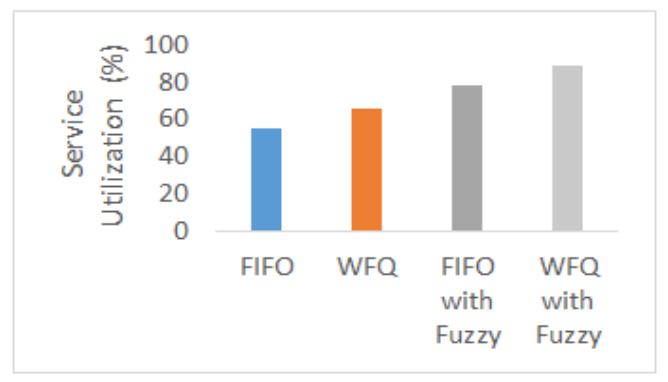

Figure 17. Service utilization for packet scheduling algorithms

Table 2 and Table 4 show results obtained from membership function values. Subsequently, IFELSE rules in Mamdani fuzzy inference system [26] are used for LSP1 and LSP2 as shwon in Table 3 and Table 5 respectively. Due to the computational complexity, four out of seven input parameters would be appropriately used. These are Throughput (T), Delay (D), Packet Loss Rate (PLR), and Utilisation Rate (UR). The theory of Union and intersection are used as the operations of fuzzy set in this respect illustrated in (4) and (5). The operation of intersection is applicable to the rows ("Min" method) in both tables. Whilst the operation of the union would be applied to the last column ("Max" method) for final membership values in the two tables. Union: 
Table 2. Membership function values LSP1)

\begin{tabular}{lccc}
\hline \multicolumn{1}{c}{ Criteria } & Low (L) & Medium $(\mathrm{M})$ & High $(\mathrm{H})$ \\
\hline Bandwidth (Bw) & 0 & 0.45 & 0.6 \\
Delay (D) & 0.2 & 0.8 & 0 \\
Cost (C) & 0.35 & 0.7 & 0 \\
Reliability(R) & 0 & 0.45 & 0.6 \\
Throughput (T) & 0 & 0.35 & 0.7 \\
Packet loss rate (PLR) & 0.25 & 0.8 & 0 \\
Utilisation rate (UR) & 0 & 0.2 & 0.85 \\
\hline
\end{tabular}

Table 3. Result of IF-ELSE (LSP1)

\begin{tabular}{cccccc}
\hline $\mathrm{T}$ & $\mathrm{D}$ & $\mathrm{PLR}$ & $\mathrm{UR}$ & Decision & Final Membership Values \\
\hline $\mathrm{M}(0.35)$ & $\mathrm{L}(0.2)$ & $\mathrm{L}(0.25)$ & $\mathrm{M}(0.2)$ & $\mathrm{PY}$ & 0.2 \\
$\mathrm{M}(0.35)$ & $\mathrm{L}(0.2)$ & $\mathrm{L}(0.25)$ & $\mathrm{H}(0.85)$ & $\mathrm{PY}$ & 0.2 \\
$\mathrm{M}(0.35)$ & $\mathrm{L}(0.2)$ & $\mathrm{M}(0.8)$ & $\mathrm{M}(0.2)$ & $\mathrm{Y}$ & 0.2 \\
$\mathrm{M}(0.35)$ & $\mathrm{L}(0.2)$ & $\mathrm{M}(0.8)$ & $\mathrm{H}(0.85)$ & $\mathrm{Y}$ & 0.2 \\
$\mathrm{M}(0.35)$ & $\mathrm{M}(0.8)$ & $\mathrm{L}(0.25)$ & $\mathrm{M}(0.2)$ & $\mathrm{PY}$ & 0.2 \\
$\mathrm{M}(0.35)$ & $\mathrm{M}(0.8)$ & $\mathrm{L}(0.25)$ & $\mathrm{H}(0.85)$ & $\mathrm{PY}$ & 0.25 \\
$\mathrm{M}(0.35)$ & $\mathrm{M}(0.8)$ & $\mathrm{M}(0.8)$ & $\mathrm{M}(0.2)$ & $\mathrm{PN}$ & 0.2 \\
$\mathrm{M}(0.35)$ & $\mathrm{M}(0.8)$ & $\mathrm{M}(0.8)$ & $\mathrm{H}(0.85)$ & $\mathrm{PN}$ & 0.35 \\
$\mathrm{H}(0.7)$ & $\mathrm{L}(0.2)$ & $\mathrm{L}(0.25)$ & $\mathrm{M}(0.2)$ & $\mathrm{Y}$ & 0.2 \\
$\mathrm{H}(0.7)$ & $\mathrm{L}(0.2)$ & $\mathrm{L}(0.25)$ & $\mathrm{H}(0.85)$ & $\mathrm{Y}$ & 0.2 \\
$\mathrm{H}(0.7)$ & $\mathrm{L}(0.2)$ & $\mathrm{M}(0.8)$ & $\mathrm{M}(0.2)$ & $\mathrm{Y}$ & 0.2 \\
$\mathrm{H}(0.7)$ & $\mathrm{L}(0.2)$ & $\mathrm{M}(0.8)$ & $\mathrm{H}(0.85)$ & $\mathrm{PY}$ & 0.2 \\
$\mathrm{H}(0.7)$ & $\mathrm{M}(0.8)$ & $\mathrm{L}(0.25)$ & $\mathrm{M}(0.2)$ & $\mathrm{PY}$ & 0.2 \\
$\mathrm{H}(0.7)$ & $\mathrm{M}(0.8)$ & $\mathrm{L}(0.25)$ & $\mathrm{H}(0.85)$ & $\mathrm{PY}$ & 0.25 \\
$\mathrm{H}(0.7)$ & $\mathrm{M}(0.8)$ & $\mathrm{M}(0.8)$ & $\mathrm{M}(0.2)$ & $\mathrm{Y}$ & 0.2 \\
$\mathrm{H}(0.7)$ & $\mathrm{M}(0.8)$ & $\mathrm{M}(0.8)$ & $\mathrm{H}(0.85)$ & $\mathrm{PN}$ & 0.7 \\
\hline
\end{tabular}

Table 4. Membership function values (LSP2)

\begin{tabular}{lccc}
\hline \multicolumn{1}{c}{ Criteria } & Low $(\mathrm{L})$ & Medium $(\mathrm{M})$ & High $(\mathrm{H})$ \\
\hline Bandwidth (Bw) & 0.6 & 0.45 & 0 \\
Delay (D) & 0.7 & 0.4 & 0 \\
Cost (C) & 0.35 & 0.7 & 0 \\
Reliability(R) & 0 & 0.45 & 0.6 \\
Throughput (T) & 0 & 0.45 & 0.6 \\
Packet loss rate (PLR) & 0.3 & 0.7 & 0 \\
Utilisation rate (UR) & 0 & 0.6 & 0.4 \\
\hline
\end{tabular}

Table 5. Result of IF-ELSE (LSP2)

\begin{tabular}{cccccc}
\hline $\mathrm{T}$ & $\mathrm{D}$ & $\mathrm{PLR}$ & $\mathrm{UR}$ & Decision & Final Membership Values \\
\hline $\mathrm{M}(0.45)$ & $\mathrm{L}(0.7)$ & $\mathrm{L}(0.3)$ & $\mathrm{M}(0.6)$ & $\mathrm{PY}$ & 0.3 \\
$\mathrm{M}(0.45)$ & $\mathrm{L}(0.7)$ & $\mathrm{L}(0.3)$ & $\mathrm{H}(0.4)$ & $\mathrm{PY}$ & 0.3 \\
$\mathrm{M}(0.45)$ & $\mathrm{L}(0.7)$ & $\mathrm{M}(0.7)$ & $\mathrm{M}(0.6)$ & $\mathrm{Y}$ & 0.45 \\
$\mathrm{M}(0.45)$ & $\mathrm{L}(0.7)$ & $\mathrm{M}(0.7)$ & $\mathrm{H}(0.4)$ & $\mathrm{Y}$ & 0.4 \\
$\mathrm{M}(0.45)$ & $\mathrm{M}(0.4)$ & $\mathrm{L}(0.3)$ & $\mathrm{M}(0.6)$ & $\mathrm{PY}$ & 0.3 \\
$\mathrm{M}(0.45)$ & $\mathrm{M}(0.4)$ & $\mathrm{L}(0.3)$ & $\mathrm{H}(0.4)$ & $\mathrm{PY}$ & 0.3 \\
$\mathrm{M}(0.45)$ & $\mathrm{M}(0.4)$ & $\mathrm{M}(0.7)$ & $\mathrm{M}(0.6)$ & $\mathrm{PN}$ & 0.4 \\
$\mathrm{M}(0.45)$ & $\mathrm{M}(0.4)$ & $\mathrm{M}(0.7)$ & $\mathrm{H}(0.4)$ & $\mathrm{PN}$ & 0.4 \\
$\mathrm{H}(0.6)$ & $\mathrm{L}(0.7)$ & $\mathrm{L}(0.3)$ & $\mathrm{M}(0.6)$ & $\mathrm{Y}$ & 0.3 \\
$\mathrm{H}(0.6)$ & $\mathrm{L}(0.7)$ & $\mathrm{L}(0.3)$ & $\mathrm{H}(0.4)$ & $\mathrm{Y}$ & 0.3 \\
$\mathrm{H}(0.6)$ & $\mathrm{L}(0.7)$ & $\mathrm{M}(0.7)$ & $\mathrm{M}(0.6)$ & $\mathrm{Y}$ & 0.6 \\
$\mathrm{H}(0.6)$ & $\mathrm{L}(0.7)$ & $\mathrm{M}(0.7)$ & $\mathrm{H}(0.4)$ & $\mathrm{PY}$ & 0.4 \\
$\mathrm{H}(0.6)$ & $\mathrm{M}(0.4)$ & $\mathrm{L}(0.3)$ & $\mathrm{M}(0.6)$ & $\mathrm{PY}$ & 0.3 \\
$\mathrm{H}(0.6)$ & $\mathrm{M}(0.4)$ & $\mathrm{L}(0.3)$ & $\mathrm{H}(0.4)$ & $\mathrm{PY}$ & 0.3 \\
$\mathrm{H}(0.6)$ & $\mathrm{M}(0.4)$ & $\mathrm{M}(0.7)$ & $\mathrm{M}(0.6)$ & $\mathrm{Y}$ & 0.4 \\
$\mathrm{H}(0.6)$ & $\mathrm{M}(0.4)$ & $\mathrm{M}(0.7)$ & $\mathrm{H}(0.4)$ & $\mathrm{PN}$ & 0.4 \\
\hline
\end{tabular}

$$
\mu_{\bar{A} \cup \bar{B}}(x)=\mu_{\bar{A}}(x) \vee \mu_{\bar{B}}(x) \text { or } \mu_{\bar{A} \cup \bar{B}}=\max \left(\mu_{\bar{A}}(x), \mu_{\bar{B}}(x)\right)
$$

Intersection:

$$
\mu_{\bar{A} \cap \bar{B}}(x)=\mu_{\bar{A}}(x) \wedge \mu_{\bar{B}} \text { or } \mu_{\bar{A} \cap \bar{B}}=\min \left(\mu_{\bar{A}}(x), \mu_{\bar{B}}(x)\right)
$$


In the IF-ELSE rules, maximum of each decision values would be considered i.e. Yes (Y), Probably Yes (PY), and Probably No (PN). According to the last column for LSP 1 table, there are six (Y), seven (PY), and three $(\mathrm{PN})$. Then, maximum decision values are: $0.2,0.25$, and 0.7 respectively. If the weightings assigned to each possibility are $\mathrm{Y}=0.61, \mathrm{PY}=0.8$, and $\mathrm{PN}=0.21$ as shown in Figure 18 (a). While the last column for LSP 2 table, there are six (Y), seven (PY), and three (PN). This is followed by maximum decision values: $0.6,0.45$, and 0.4 respectively. If the weightings assigned to each possibility are $\mathrm{Y}=0.8, \mathrm{PY}=0.76$, and $\mathrm{PN}=0.38$ as shown in Figure 18 (b). The scheduling of packets among selected paths indicate decisionmaking problem in a fuzzy network environment. Therefore, LSP2 outperforms than LSP1 in terms of service satisfaction with an increase of 28\%. Figures 19 (a), 19 (b) and Figures 20 (a), 20 (b) illustrate the fuzzy LSP selection for certain fuzzy rules in the fuzzy logic controller using different fuzzy input variables on the $\mathrm{x}$ and $\mathrm{y}$-axis of the 3-D graph respectively. These figures show how the value of service-aware LSP selection factor varies with respect to different input parameters into the fuzzy controller, which is the main fuzzy logic system.

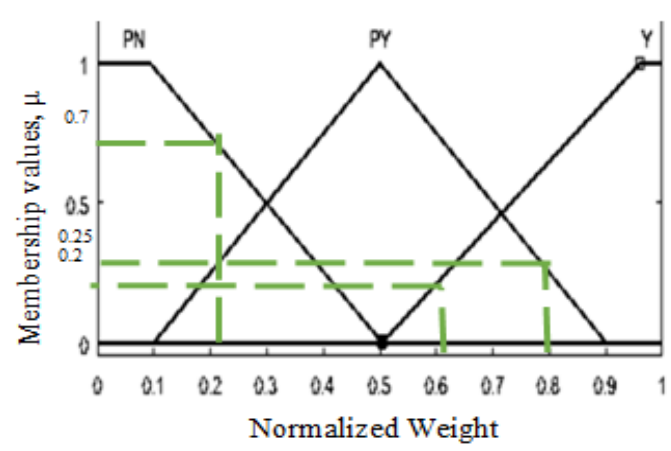

(a)

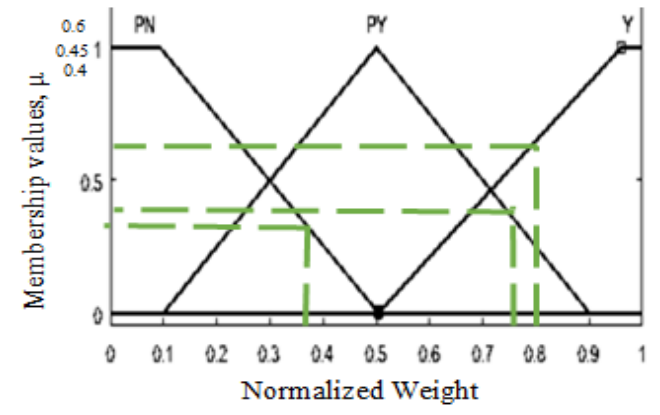

(b)

Figure 18. Normalized weight of membership values

Service satisfaction factor for LSP1 $=\frac{(0.2 \times 0.61)+(0.25 \times 0.8)+(0.7 \times 0.21)}{0.2+0.25+0.7}=0.39$

Service satisfaction factor for LSP2 $=\frac{(0.6 \times 0.8)+(0.45 \times 0.76)+(0.4 \times 0.38)}{0.6+0.45+0.4}=0.67$

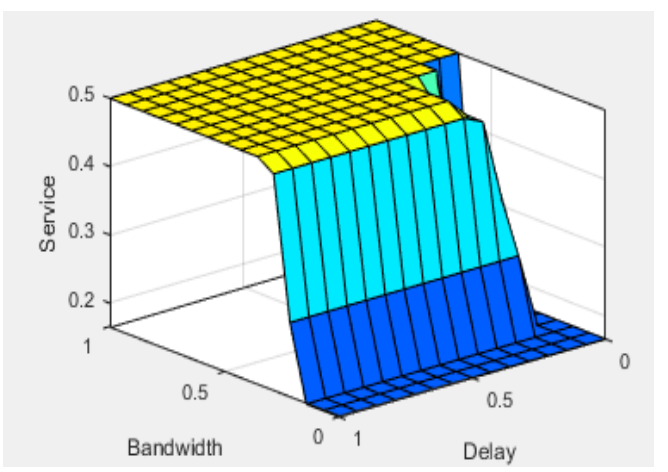

(a)

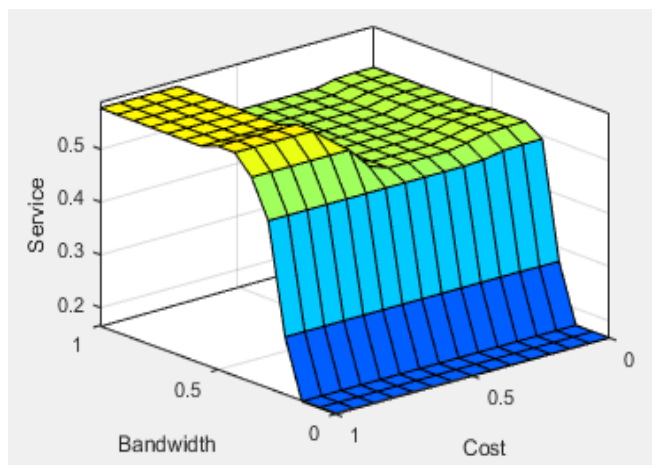

(b)

Figure 19. These figure are; (a) Bandwidth versus delay (b) Bandwidth versus cost 


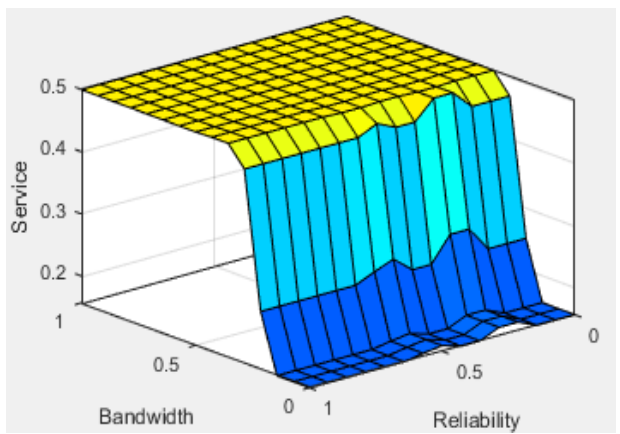

(a)

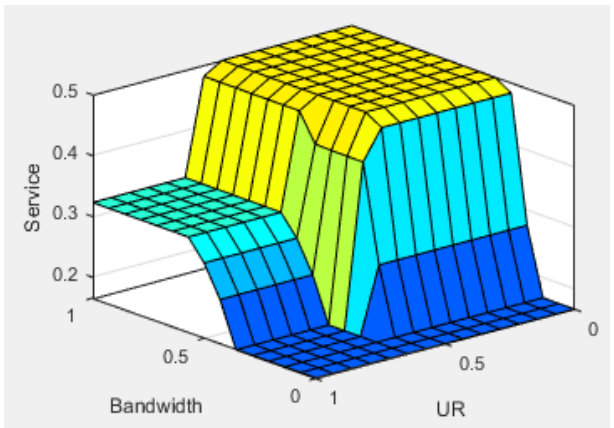

(b)

Figure 20. These figure are; (a) Bandwidth versus reliability, (b) Bandwidth versus UR

\section{CONCLUSION}

In conclusion, the PPA with the incorporation of FPSA in the IP over MPLS networks had been designed in this research work. Then, the bandwidth management algorithm had been easily applied to the aspect of traffic engineering by using its functions such as the establishment of explicit route for the LSP Tunnel, which are already shown. It is observed that MPLS performed better than IP with the conventional methods of routing and switching using non real-time traffic, which is justified in the simulation results. Furthermore, the fuzzy based service-aware LSPs for forwarding scheduled packets are obtained from computation to avoid the situations of underutilization and overutilization of the paths. Therefore, FPSA has a significant impact on making the decision for the selection of satisfactory service. However, fuzzy lacks the provision for solving complexity of multiple input parameters. Further work can be extended to solve the problem of dimensionality in the fuzzy system for multiple input parameters in IP over MPLS networks as well as the introduction of real-time traffic.

\section{ACKNOWLEDGEMENTS}

The corresponding author wish to express profound appreciation to his sponsor Tertiary Education Trust Fund (TETFUND) for this immense opportunity to undergo the PhD programme.

\section{REFERENCES}

[1] Divya Sharma, Renu Singla, “A Detail Review on Multiprotocol Label Switching (MPLS)," International Journal of Advanced Research in Computer Engineering \& Technology IJARCET, vol. 3, no. 2, pp. 354-361, 2015.

[2] V. Foteinos, K. Tsagkaris, P. Peloso, L. Ciavaglia, P. Demestichas, "Operator-Friendly Traffic Engineering in IP/MPLS Core Networks," in IEEE Transactions on Network and Service Management, vol. 11, no. 3, Sept 2014, pp. 333-349.

[3] A. Sydney, J. Nutaro, C. Scoglio, D. Gruenbacher, N. Schulz, "Simulative Comparison of Multiprotocol Label Switching and OpenFlow Network Technologies for Transmission Operations," in IEEE Transactions on Smart Grid, vol. 4, no. 2, pp. 763-770, June 2013.

[4] O. Z. Mustapha, M. Ali, Y. F. Hu, R. A. Abd-Alhameed, "Fuzzy Based Packet Scheduling Scheme using Non-Real Time Traffic in IP/MPLS Networks," 2019 7th International Conference on Future Internet of Things and Cloud (FiCloud), Istanbul, Turkey, pp. 385-392, 2019.

[5] O. Z. Mustapha, Y. F. Hu, R. A. Abd-Alhameed, H. S. Abdullahi, "Approach to Label Distribution Protocol Signaling Using Multimedia Services for Bandwidth Allocation," 2018 UKSim-AMSS 20th International Conference on Computer Modelling and Simulation (UKSim), Cambridge, 2018, pp. 157-162.

[6] G. R. Ash, "Traffic Engineering and QoS Optimization of Integrated Voice \& Data Networks," Morgan Kaufmann, 2006.

[7] T. Issariyakul, E. Hossain, "Introduction to Network Simulator 2 (NS2)," in Introduction to Network Simulator NS2, ed: Springer, pp. 1-18, 2009.

[8] Reyadh Shaker Naoum, Mohanand Maswady, "Performance Evaluation for VOIP over IP and MPLS," World of Computer Science and Information Technology Journal, vol. 2, no. 3, pp. 110-114, 2012.

[9] J. Barakovic, H. Bajric, A. Husic, "Multimedia Traffic Analysis of MPLS and non-MPLS Network," Proceedings ELMAR 2006, Zadar, Croatia, 2006, pp. 285-288.

[10] M. K. Porwal, A. Yadav, S. V. Charhate, "Traffic Analysis of MPLS and Non MPLS Network including MPLS Signaling Protocols and Traffic Distribution in OSPF and MPLS," 2008 First International Conference on Emerging Trends in Engineering and Technology, Nagpur, India, 2008, pp. 187-192. 
[11] M. Khan, "MPLS Traffic Engineering in ISP Network," International Journal of Computer Applications, vol. 59, no. 4, pp. 23-32, 2012.

[12] O. Z. Mustapha, R. E. Sheriff, F. L. C. Ong, "Bandwidth Management Using MPLS Model for Future Mobile Wireless Networks," International Conference on Wireless and Satellite Systems, Oxford UK, Springer, 2017, pp. 62-71.

[13] S. Kulkarni, R. Sharma, I. Mishra, "New QoS routing algorithm for MPLS networks using delay and bandwidth constraints," International Journal of Information and Communication Technology Research, vol. 2, no. 3, pp. 285293, 2012.

[14] C. Rosen, A. Viswanathan, R. Callon, "Multiprotocol Label Switching Architecture," IETF RFC 3031, pp. 1-61, Jan. 2001.

[15] A. V. Lemeshko, Ahmad M. Hailan, Zaid A. Sabeeh, "Mathematical Model of MPLS-Network Supporting Traffic Engineering DiffServ," International Journal of Wisdom Based Computing, vol. 1, no. 3, pp. 119-122, Dec 2011.

[16] A. Albdoor, G. Kannan, "Analysis of MPLS and IP Networks Performance to Improve the QoS using Opnet Simulator," Journal of Emerging Trends in Computing and Information Sciences, vol. 8, no. 1, 2017.

[17] D. Awduche, A. Chiu, A. Elwalid, I. Widjaja, X. Xiao, "Overview and Principles of Internet Traffic Engineering," IETF Internet Draft-IETF-tewg-principles, pp. 1-71, 2002.

[18] D. Kalav, S. Gupta, "Congestion Control in Communication Network Using RED, SFQ and REM Algorithm," International Refereed Journal of Engineering and Science IRJES, vol. 1, no. 2, pp 41-45, 2012.

[19] L. Crawford, Marshall, "A Dynamic Fast Packet Scheduling Algorithm For Open And Programmable Networks," The Seventeenth UK Teletraffic Theory Symposium, Citeseerx, 2001.

[20] Gaeil Ahn, Woojik Chun, "Design and implementation of MPLS network simulator supporting LDP and CR-LDP," Proceedings IEEE International Conference on Networks 2000 ICON 2000, Networking Trends and Challenges in the New Millennium, Singapore, 2000, pp. 441-446.

[21] Sarhan M. Musa, Mahamadou Tembely, Matthew N. O. Sadiku, Pamela Obliomon, "Comparative Study of Different Queuing Scheduling Disciplines," International Journal of Engineering Research and Applications, vol. 3, no. 6, pp. 1587-1591, 2013.

[22] P. M. L. Chan, R. E. Sheriff, Y. F. Hu, P. Conforto, C. Tocci, "Mobility management incorporating fuzzy logic for heterogeneous a IP environment," in IEEE Communications Magazine, vol. 39, no. 12, pp. 42-51, Dec. 2001.

[23] M. N. Soorki, H. Rostami, "Label switched protocol routing with guaranteed bandwidth and end to end path delay in MPLS networks," Journal of Network and Computer Applications, vol. 42, pp. 21-38, 2014.

[24] S. Kharb, A. Singhrova, "Fuzzy based priority aware scheduling technique for dense industrial IoT networks," Journal of Network and Computer Applications, vol. 125, pp. 17-27, 2019.

[25] O. A. Egaji, A. Griffiths, M. S. Hasan, Hong-Nian Yu, "A Comparison of Mamdani and Sugeno Fuzzy BasedPacket Scheduler for MANET with a Realistic WirelessPropagation Model," International Journal of Automation and Computing, vol. 12, no. 1, pp. 1-13, 2015.

[26] P. Neamatollahi, M. Naghibzadeh, S. Abrishami, "Fuzzy-Based Clustering-Task Scheduling for Lifetime Enhancement in Wireless Sensor Networks," in IEEE Sensors Journal, vol. 17, no. 20, pp. 6837-6844, 2017.

\section{BIOGRAPHIES OF AUTHORS}
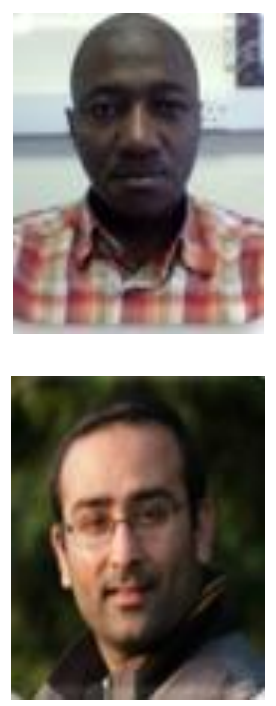

Oba Zubair Mustapha received his both Bachelor and Master of Engineering in Electrical Engineering from University of Ilorin, Nigeria, in 1997 and 2011 respectively. He possessed his doctor of philosophy $(\mathrm{PhD})$ from University of Bradford, United Kingdom, in 2020. Worked as a System Engineer at Cyberaccess \& Communication Ltd Nigeria, from 2000 to 2003. He then joined Kwara State Polytechnic, Institute of Technology, Nigeria, started with Lecturer II and now Senior Lecturer in the department of Electrical/Electronic Engineering from 2007 till date. His area of research interest are in the field of Electronics and Telecommunications, Communications Networks, Network Protocols, Radio Resource Management, and Artificial Intelligence. He is member of COREN (Registered Engineer), IAENG, and IEEE.

Muhammad Ali has (Bachelor of Science) degree in computer science in 2006 from Comsats University Islamabad Pakistan. Then completed MSc and $\mathrm{PhD}$ in Mobile \& Satellite Communications in 2007 and 2013 from University of Bradford United Kingdom. Worked as Research Assistant in Future Ubiquitous Networks Lab at University of Bradford UK, from 2011 to 2015. In 2016 worked as a Lecturer in Department of Electrical Engineering and Computer Science, University of Bolton. Also worked as lead software developer for a London based software house "Direct2Success" from 2016 to 2018. Currently working as a Post-Doctoral Research Staff at Future Ubiquitous Networks Lab, Faculty of Engineering and Informatics, University of Bradford UK from 2018. Acquired wide range of skills set while working on multiple EU/Innovative-UK/Industrial funded research and development projects in the field of avionics communications networks, heterogeneous wireless networks, software defined networks, artificial intelligence, embedded systems, network security and desktop application development. 


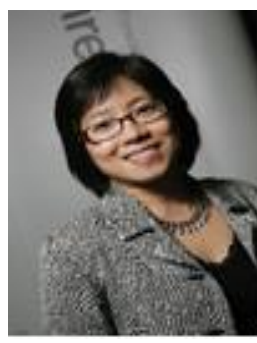

Yim Fun $\mathrm{Hu}$ is Professor of Wireless Communications Engineering at the University of Bradford. She received the B.Sc.(Hons.) degree in Mathematical Sciences and Ph.D. degree in Information Systems Engineering, both from the University of Bradford, U.K. Professor Hu has more than 25 years research experience in mobile, wireless and satellite communications technologies with applications to aeronautical communications, high speed trains communications, Internet of Things, future cities, and smart health. She is a Principle Investigator of many flagship projects funded by the European Union, European Space Agency, U.K., and other international funding councils. She published more than 150 international peer reviewed journals and conference paper. She is a member of the UK EPSRC peer review college, and a Fellow of the Institute of Engineering and Technology.

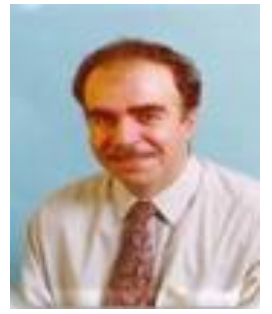

Raed A. Abd-Alhameed is Professor of Electromagnetic and Radio Frequency Engineering at the University of Bradford, UK. He has long years' research experience in the areas of Radio Frequency, Sensor Design, Signal Processing, Propagations, Antennas and Electromagnetic Computational Techniques, and has published over 600 academic journal and conference papers; in addition, he is co-authors of four books and several book chapters. At the present he is the leader of Radio Frequency, Antennas, Propagation, sensor design and Signal Processing; in addition to leading the Communications research group for years within the School of Engineering and Informatics, Bradford University, UK. He is Principal Investigator for several funded applications to EPSRCs and leader of several successful knowledge Transfer Programmes. He has also been a co-investigator in several funded research projects including 1) H2020 MARIE Skłodowska-CURIE ACTIONS: Innovative Training Networks (ITN) "Secure Network Coding for Next Generation Mobile Small Cells 5G-US", 2) Nonlinear and demodulation mechanisms in biological tissue (Dept. of Health, Mobile Telecommunications \& Health Research Programme and 3) Assessment of the Potential Direct Effects of Cellular Phones on the Nervous System 